\title{
O uso terapêutico da cetamina na depressão resistente ao tratamento: uma revisão bibliográfica
}

\section{The therapeutic use of ketamine in treatment-resistant depression: a literature review}

\author{
Ana Clara Abreu Lima de Paula \\ Instituição: Universidade Federal de Juiz de Fora (UFJF), Juiz de Fora - MG; \\ Carolina Lopes de Araújo \\ Universidade Federal de Minas Gerais (UFMG), Belo Horizonte - MG; \\ Mariana Codevilla Santana de Moura \\ Universidade Federal de Ouro Preto (UFOP), Ouro Preto - MG; \\ Leonardo de Paula Junqueira \\ Hospital São Paulo (CCMHSP), Muriaé - MG.
}

\section{RESUMO}

Palavras-chave: Cetamina, Depressão, Tratamento.

\section{ABSTRACT}

Keywords: Ketamine, Depression, Treatment.

\section{INTRODUÇÃO}

O transtorno depressivo maior (TDM) é uma doença neuropsiquiátrica crônica, altamente prevalente, tida como a principal causa de incapacidade funcional no mundo (LACERDA ALT, 2020; HASHIMOTO K, 2020). Seus sintomas envolvem sensações persistentes de tristeza, desinteresse, raiva e desânimo (LENT JK, et al., 2019). A fisiopatologia da doença é heterogênea, sendo a hipótese mais aceita a das monoaminas, a qual propõe que a neurotransmissão e cognição no TDM seriam prejudicadas devido à menor disponibilidade de neurotransmissores monoaminérgicos no cérebro (LACERDA ALT, 2020).

A depressão resistente ao tratamento (DRT) ocorre quando pelo menos dois fármacos de intervenção tradicional apresentam resposta insatisfatória em quadros de TDM. Nesses casos, é possível recorrer à terapia eletroconvulsiva e à estimulação magnética transcraniana, porém, alguns pacientes podem não responder bem a essas opções (FRANCO FM, et al., 2020). Dessa forma, a cetamina surge como alternativa terapêutica para esse transtorno.

A cetamina é usada como analgésico e anestésico desde 1970 e, nas últimas décadas, doses subanestésicas dessa medicação têm mostrado efeito antidepressivo e anti-suicida em pacientes com 
depressão, unipolar ou bipolar, resistentes ao tratamento. Seu uso é considerado seguro e satisfatório, pois seus efeitos colaterais mais relatados são transitórios (IQBAL SZ e MATHEW SJ, 2020).

\section{OBJETIVO}

Revisar e sintetizar os conhecimentos recentes da literatura científica sobre o uso da cetamina no tratamento da depressão, visando esclarecer questionamentos acerca do uso dessa terapia, seus mecanismos de ação, formas de administração, segurança, eficácia, tolerabilidade, riscos e efeitos adversos.

\section{REVISÃO BIBLIOGRÁFICA}

A cetamina apresenta alta afinidade para o sítio da fenciclidina do receptor glutamatérgico N-metil-Daspartato (NMDAR), caracterizando-se como forte antagonista do mesmo. Seu mecanismo de ação facilita a sinaptogênese e potenciação sináptica e, além dos efeitos nos sistemas glutamato-GABA, também pode ter impactos relevantes em outros alvos na DRT, como receptores colinérgicos e opióides (MCINTYRE RS, et al., 2021).

Ensaios clínicos com a cetamina estabeleceram sua rápida eficácia no tratamento do TDM e DRT. Na injeção intravenosa (IV) da mistura racêmica de cetamina, a eficácia de curto prazo sugere que para manter o efeito terapêutico é necessário repetir a aplicação periodicamente. As doses de $0.5 \mathrm{mg} / \mathrm{kg}$ e $1.0 \mathrm{mg} / \mathrm{kg}$ têm maior eficácia que doses inferiores (FAVA M, et al., 2020). Além disso, foram observados efeitos antisuicidas 40 minutos após a infusão, que perduram por tempo variável, geralmente cerca de 7 dias após a administração prévia (IQBAL SZ e MATHEW SJ, 2020).

Nas horas seguintes à infusão, é possível que o paciente tenha efeitos colaterais como tontura, sonolência, náusea, dor de cabeça e visão turva, os quais são temporários e proporcionais à dosagem, tendendo a passar com a descontinuação da infusão (FRANCO FM, et al., 2020). Efeitos colaterais clinicamente mais significativos tendem a aparecer apenas em casos de uso prolongado ou sob uso de doses altas (IQBAL SZ e MATHEW SJ, 2020).

A escetamina intranasal, combinada com antidepressivos convencionais, mostrou ter ação rápida e eficaz nos pacientes (PAPAKOSTAS GI et al, 2020) devido à grande vascularização da região. Porém, não revela resultados mais eficazes aumentando a dose ou o número de aplicações (IQBAL SZ e MATHEW SJ, 2020). As evidências sobre a eficácia das outras formas de administração da cetamina na depressão, como a oral, sublingual, subcutânea e intramuscular, ainda são insuficientes (MCINTYRE RS, et al., 2021). 


\section{DISCUSSÃO}

A cetamina como antidepressivo demonstra efeito rápido, porém pouco duradouro, segurança e tolerabilidade satisfatórias a curto prazo, com efeitos adversos transitórios. Todavia, a monitorização do paciente durante sua aplicação, com estabelecimento e equipe profissional adequados, é indispensável.

Em 2019, a escetamina intranasal combinada com antidepressivos orais, foi aprovada pelo Food and Drug Administration (FDA) para tratamento de adultos com DRT e, em 2020, adultos com TDM e ideações suicidas (MCINTYRE RS, et al., 2021). No Brasil, em novembro de 2020, a Agência Nacional de Vigilância Sanitária (Anvisa), também aprovou o spray nasal para tratamento da DRT em adultos (ANVISA, 2020).

Apesar dos avanços científicos, os conhecimentos sobre eficácia, segurança e tolerabilidade a longo prazo da cetamina são limitados (MCINTYRE RS, et al., 2021). Uma alternativa é desenvolver antidepressivos com mecanismos de ação semelhantes ao da cetamina e efeito rápido sobre o sistema nervoso (NA KS e KIM YK, 2020).

\section{CONSIDERAÇÕES FINAIS}

Sendo assim, com a ampliação dos conhecimentos sobre os efeitos antidepressivos da cetamina e a aprovação da escetamina intranasal pelos órgãos reguladores, consensos científicos acerca da padronização do tratamento de transtornos psiquiátricos com essa substância são essenciais para a prática clínica segura. Também é necessária a realização de grandes ensaios controlados randomizados comparativos entre as formas de administração e, ainda, sobre eficácia, segurança e tolerabilidade em tratamentos de longo prazo e em populações pediátricas e geriátricas. Além disso, deve-se desenvolver estratégias para prolongar a eficácia da cetamina nos pacientes com DRT. 


\section{REFERÊNCIAS}

1. BRASIL. Agência Nacional de Vigilância Sanitária. 2020. Disponível em: https://consultas.anvisa.gov.br/\#/documentos/tecnicos/25351068398201941/. Acessado em: 01 de maio de 2021.

2. FAVA M, FREENAB MP, FLYNN M, et al. Double-blind, placebocontrolled, dose-ranging trial of intravenous ketamine as adjunctive therapy in treatment-resistant depression (TRD). Molecular Psychiatry, 2020; 25(7): 1592-1603.

3. FRANCO FM, LIMA AJM, ALVES NC, et al. Os efeitos do uso da cetamina em pacientes com depressão resistente ao tratamento. Brazilian Journal of Development, 2020; 6(6): 36999-37016.

4. HASHIMOTO K. Molecular mechanisms of the rapid-acting and long-lasting antidepressant actions of (R)-ketamine. Biochemical Pharmacology, 2020; 177: 113935.

5. IQBAL, SZ e MATHEW, SJ. Ketamine for depression clinical issues. Advances in Pharmacology, 2020; 89: 131-162.

6. LACERDA, ALT. Esketamine/ketamine for treatment-resistant depression. Brazilian Journal of Psychiatry, 2020; 42(6): 579-580.

7. LENT JK, ARREDONDO A, PUGH MA, et al. Ketamine and Treatment-Resistant Depression. American Association of Nurse Anesthetists Journal, 2019; 87(5): 411-419.

8. MCINTYRE RS, ROSENBLAT JD, N.EMEROFF CB, et al. Synthesizing the evidence for ketamine and esketamine in treatment-resistant depression: an international expert opinion on the available evidence and implementation. American Journal of Psychiatry, 2021;

9. NA, KS; KIM, YK. Increased use of ketamine for the treatment of depression: Benefits and concerns. Progress in Neuro-Psychopharmacology and Biological Psychiatry, 2020; 104: 110060.

10. PAPAKOSTAS GI, SALLOUM NC, HOCK RS, et al. Efficacy of esketamine augmentation in major depressive disorder: a meta-analysis. Journal of Clinical Psychiatry, 2020; 81(4): 19r12889. 